\title{
The Process of Formation the Autoimmune Reaction in the Gastric Mucosa the Damaged Liver
}

\author{
Trubitsyna $\mathbf{I}^{1 *}$, Vinokurova $\mathrm{L}^{1}$, Tarasova $\mathrm{T}^{2}$, Lesko K1, Dorofeev $\mathrm{A}^{1}$, Papysheva $\mathrm{L}^{1,3}$, Efremov ${ }^{1}$, Abdulatipova $\mathrm{Z}^{1}$, \\ Smirnova $\mathbf{A}^{1,4}$ and Varvanina $\mathrm{G}^{1}$ \\ ${ }^{1}$ Moscow clinical research and practice center Of the Moscow Department of health, Russia \\ ${ }^{2}$ Professor of the Department of psychology of the Historical and sociological Institute of the Federal state budgetary educational institution of higher \\ education, Ogarev Mordovian state University, Russia \\ ${ }^{3}$ Central research Institute of epidemiology of Rospotrebnadzor, Russia \\ ${ }^{4}$ Federal state budgetary institution "national medical research center of Oncology named after N. N. Blokhin" of the Ministry of health of the Russian \\ Federation, Russia
}

Submission: September 06, 2020; Published: October 05, 2020

*Corresponding author: Trubitsyna I, Moscow clinical research and practice center of the Moscow Department of health, Moscow, Russia

Keywords: Autoimmune component; Liver; Mucous membrane; Gastroduodenal ulcer

\section{Introduction}

The presence of portal hypertension hinders the outflow of blood from the gastric mucosa, causing venous congestion and hypoxia of the mucosa [1]. A decrease in synthetic liver function disrupts the metabolism of 5-HT, maintaining its high content in the blood, which in turn reduces the volume of flowing blood, causing stasis in the capillaries and venules. Disrupting the architectonics of the microcirculatory bed of the gastric mucosa $5-\mathrm{HT}$, reduces its protective properties. At the same time, the aggressive properties of gastric juice are increased by rearranging the spectrum of proteolytic enzymes according to the "ulcerative" type. Conclusion: cirrhosis of the liver contributes to a violation of the metabolism of 5-HT, portal hypertension hinders the outflow of blood from the stomach, causing hypoxia of the gastric mucosa, which is enhanced by the action of 5-HT on the microcirculatory bed of the mucosa [2]. A decrease in the protective properties of the mucous membrane is combined with an increase in the aggressive properties of gastric juice, which as a result contributes to the formation of ulcers and erosions in the gastric mucosa [3]. The incidence of hepatogenic gastropathy in cirrhosis of the liver was observed in 50-60 \%. The incidence of peptic ulcer disease in patients with cirrhosis is 2-6 times higher than without cirrrosis. Clinicians have noticed a link between cirrhosis of the liver and the development of pathology of the stomach and duodenum [4]. In 1937, Eppinger conducted experiments on prisoners in Dachau and described changes in the stomach and small intestine mucosa in cirrhosis of the liver. The duration of each experiment was from 6 to 12 days. Eppinger associated these changes with the effect on the mucosa of toxic metabolites coming from the gut or with factors of aggression in the stomach (acid, enzymes) [5]. In more recent times, it has been suggested that gastropathies are associated with impaired blood flow, but there is no consensus until now. In 1946. Jahn coined the term "hepatogenic ulcer" after analyzing a large number of autopsy protocols and described a clear etiopathogenetic relationship between liver cirrhosis and gastroduodenal ulceration. However, pathogenetic relationships have not been elucidated. To identify the mechanisms involved in damage to the mucous membrane (co) of the gastroduodenal zone requires experimental studies. Purpose of the first series of studies (I). To establish a causal link between the damage to the liver and the gastroduodenal area.

\section{Material and Methods}

An alcoholic experimental model of liver damage was used in 10 white Wistar rats weighing 180-220 g. The animals were 
divided into 2 groups of 5 animals in each group, the study duration is 6 days. Group 1 - D) - control (K) animals that are on the usual food and water regime; group 2-rats had two drinkers, one with a low-alcohol drink (SN), the second with water. SN was given without restrictions, as much as the rats "desired", $500 \mathrm{ml}$. Theanimals were removed from the experiment for 6 days. After the autopsy, the abdominal organs, liver and mucous membrane (MM) of the gastroduodenal zone were examined. Macro-and microphotographs have shown that against the background of liver damage, according to the main theory, endothelial cells and sinuses are damaged during the development of the disease, and blood flow obstruction at the sinusoidal level develops hepatocellular necrosis. Hepatocellular necrosis occurs as a result of both the direct toxic effect of alcohol and a violation of the blood supply to the organ (Figures 1 \& 2).

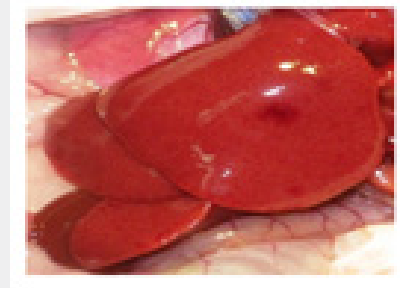

A

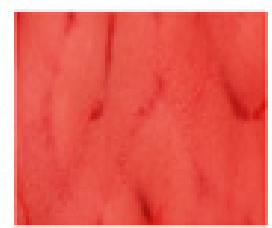

B

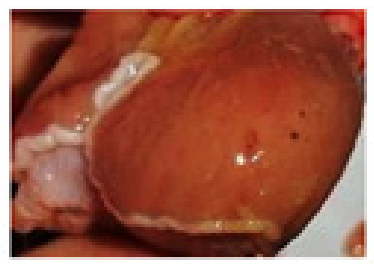

C

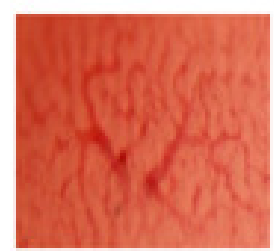

$\mathrm{D}$

Figure 1: Magnifier Macrophotographs a) Liver, subcapsular hemorrhages b) Expanded flowing capillaries $x$ 50, C: Mucous membrane stomach ulcer $\times 10, c)$ Microcirculatory disorders, dilated outflowing capillaries and a collection venule the body of the stomach $\mathrm{x} 20$.

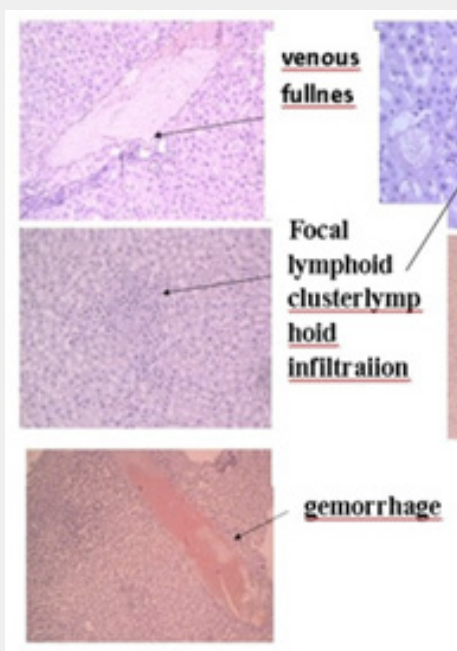

Figure 2: Liver, micrographs of liver damage G\&E x 240.

Macro-and microphotographs have shown that against the background of liver damage, according to the main theory, the development of the disease causes damage to the endothelial cells of the sinuses, obstruction of blood flow at the sinusoidal level, and hepatocellular necrosis. Hepatocellular necrosis occurs as a result of the direct toxic effect of alcohol, a violation of the blood supply to the organ. The process of dying off hepatocytes is accompanied by the death and decay of hepatocytes. Cell death occurs locally, with the destroyed parenchyma forming a clearly limited necrotic focus with lymphoid infiltration. Thus, the autoimmune component appears when hepatocytes die. antibodies are formed on various organoids and cell inclusions (mitochondria, cell wall, nucleus, etc.) Figure 2. To exclude the direct effect of alcohol on
MM, a second series of studies was conducted, with preliminary immunization of animals. The process of dying off hepatocytes is accompanied by the death and decay of hepatocytes. Cell death occurs locally, with the destroyed parenchyma forming a clearly limited necrotic focus with lymphoid infiltration. Thus, the autoimmune component appears when hepatocytes die. antibodies are formed on various organoids and cell inclusions (mitochondria, cell wall, nucleus, etc.). To exclude the direct effect of alcohol on MM, a second series of studies was conducted, with preliminary immunization of animals. The immune system is a combination of protective and damaging mechanisms that have been forming for more than 400 million years. The main task of the immune system is to protect the body from foreign and harmful 
substances of microorganisms, toxins and malignant cells. The immune system of the gastrointestinal mucosa (MALT-sistem) [4] plays a huge role in the development, maintenance and regulation of immune homeostasis [1], being an important component of the multicomponent immune system. Under physiological conditions, the functioning of the immune system, its reactivity, both systemic and local, is genetically programmed, and locally determined by the balanced production of regulatory cytokines by type 1 and type 11 T-helpers. T-cells (T-lymphocytes) that are part of the immune surveillance system must be able or learn to recognize substances that do not belong to the body. Violation of the cytokine balance plays a significant role in the chronization and progression of diseases of the digestive system. The intensity of local and systemic pathological processes depends on the intensity of the response to the volume of damage in the pathology of the stomach, gallbladder, small and large intestine, accompanied by changes in the content of cytokines in the peripheral blood and damaged tissue adjacent to the area of inflammation, providing the intensity of the local immune response. A large number of antigen- presenting cells can be found in the area between the follicle and the follicle-related intestinal epithelium (the "dome" area). The epithelium of the dome is characterized by the presence of so-called microscellular cells (M-cells), which have numerous microscells (not microvilli) on the epithelial surface and specialize in transporting antigens. The contents of the cells are separated from the environment by a biomembrane. Inside the cell is filled with cytoplasm, where various organoids and cellular inclusions are located, as well as genetic material in the form of a DRA molecule.

Each of the cell organelles performs a specific function, and together they determine the functioning of cells in General, Despite the fact that in the evolution of the reduced damaging effect on body tissues of the host, but not on endogenous substances which become "strangers" when injected into the blood or extracellular fluids. The autoimmune component itself does not cause the disease but complicates the course of an existing disease. The cause of autoimmunity are poorly understood, due to the fact that numerous reactions to corruption are intertwined, making it difficult to study them in pure form and, thus, experimental studies in intact animals allows to identify an individual or a set of elements is pathogenetically linked to ensure or prevent the development of damage to the digestive organs. This situation is determined by many factors. Initially, we can assume that this is the result of changes in regulatory mechanisms and not only. We assume that hepatitis provokes an autoimmune reaction in the duodenum, which requires proof of a causal relationship. Understanding these injuries can be established only when conducting experimental studies on healthy animals Purpose: in the experiment to trace the development of specific autoimmune reactions, consisting of increased immune sensitivity and immunological tolerance, organ hepatoduodenal area of the gastrointestinal tract.
Autoimmune reactions directed against the own subcellular structures of the membrane, cytoplasm, and nucleus play an important role in the pathogenesis of such liver lesions as autoimmunehepatitisand primary biliary cirrhosis. These diseases are accompanied by a pronounced mononuclear infiltration of portal tracts, a sharp increase in serum levels of gamma globulins, immunoglobulins, immune complexes, autoantibodies to subcellular structures-membranes, mitochondria, microsomes, nuclear components. Determine the etiological factor, the intensity of infectious, inflammatory, autoimmune processes, the activity of the disease, its prognosis, as well as the effectiveness of therapy for diseases of the small and large intestine

White rats of the Wistar line are used. The animals were divided into 6 groups of five animals each. Using a standard technique, animals were immunized with allogeneic tissues. It is known that antibodies are formed mainly to antigens that reflect the specific features of these tissues. Experiments were performed on white rats, both sexes, weighing 180-220 g (average weight $200 \mathrm{~g}$ ). The animals were kept in the vivarium in accordance with sanitary regulations. All experiments were conducted in accordance with the rules of humane treatment of animals, manipulations were performed under General anesthesia in compliance with the requirements of asepsis and antiseptics. The formation of antibodies depends on the duration of immunization and the total dose of the administered antigen. In our study, rats were immunized according to the standard scheme: tissue homogenate prepared at the rate of $1 \mathrm{~g}$ of tissue in $10 \mathrm{ml}$ of saline solution was used. Did 4 intraperitoneal injections of $2 \mathrm{ml}$ of homogenate containing $30 \mathrm{mg}$ of protein per injection, carried out 3 days later on the fourth. According to this scheme, antibodies are obtained in the titer 1: $160-1: 640$. once in the body, the foreign antigen is absorbed by macrophages, lymphocytes, and reticular cells, which turn into plasma cells that produce antibodies. The initial reaction between the antigen and the corresponding cells takes only a few minutes. A tight connection is formed between the antigen molecules and specific cell membrane receptors. In order for the process of antibody formation to begin, the antigen must penetrate $1,000,000-100,000,000$ cells. One cell should contain from 100 to several 1000 antigen molecules. When an antigen is first injected into an animal that has never been injected before, there is a certain latent period between the time of the antigen injection and the time of the appearance of antibodies in the animal's blood. The duration of the latent period depends on the properties and quantity of the antigen, the method of its administration, and the individual properties of the immunized animal. As a rule, the first antibodies are detected in the animal's blood 3-5 days after the antigen injection and reach the highest titer by $9-12$ days. After this, the antibody titer drops noticeably, but sometimes remains at a high level for many years. Taking into account that the autoimmune reaction reaches its peak after day 10 , the animals were removed from the experiment on the second day after the last injection. Study design: Group 1 control, intact 
animals (without the introduction of homogenates), administered $2.0 \mathrm{ml}$ of saline solution. $2 \mathrm{nd}$ and further experimental groups of 5 animals: with the introduction of $2 \mathrm{ml}$ of homogenate: 2 nd group: introduced homogenate of the fundal gastric mucosa. 3-I-antral gastric homogenate, 4-I-duodenal mucosal homogenate (Figure 3-6), 5--liver homogenate (Figure 5).

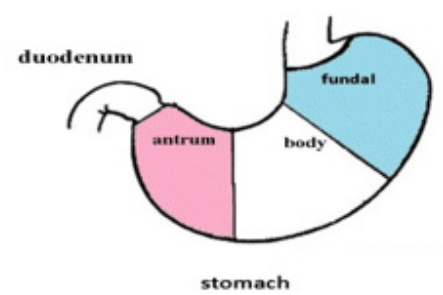

Figure 3: Diagram of anatomical zones of the stomach.
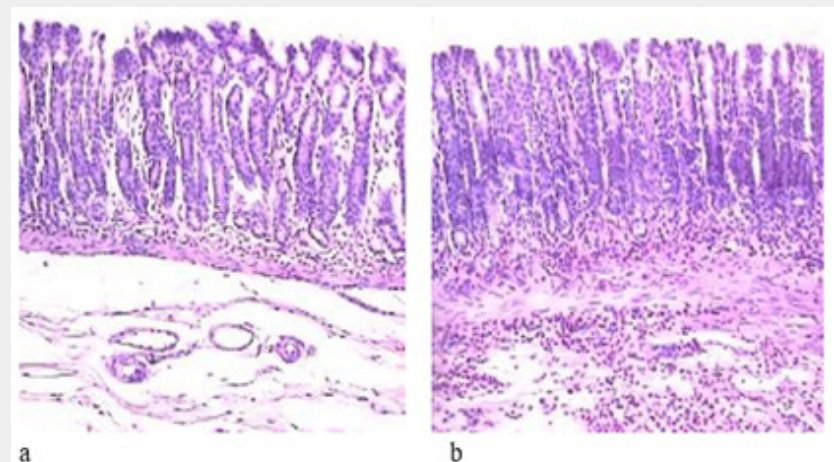

Figure 4a \& b: Antrun stomach. coloring h \& e a) Pronounced edema in the submucosal layer, weak infiltration by lymphoid cells and eosinophils in the mucous membrane. $\times 180$. b) In its own, muscular plate of the mucous membrane and submucosal layer, moderate infiltration by lymphoid cells and eosinophils. $\mathrm{x} 180$.
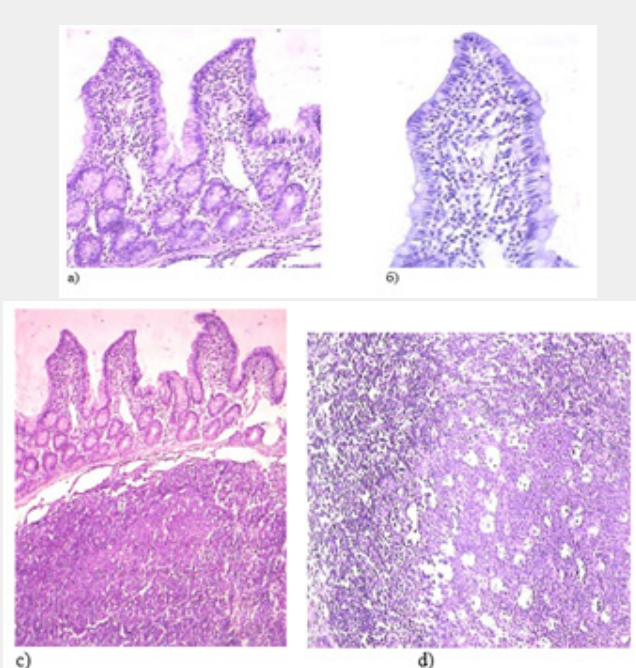

Figure 4a-d: Duodenum. Coloring h \& e. Morphological examination in all the studied parts of the stomach, duodenum and liver, hyperplasia of lymphoid tissue was observed. The lymphoid tissue of the digestive system allows us to distinguish three main groups in it: lymphocytes-lymphocytes localized in the epithelium and plasma cells that diffusely "populate" their own plate of the mucous membrane; lymphoid follicles located in the basal parts of the mucous membrane. At the same time, the weight and size of the spleen and thymus are increased. a) In the mucous membrane, moderate infiltration by lymphoid cells and eosinophils, single lymphangiectasias. $\times 250$. b) Moderate infiltration of the intestinal villi epithelium with lymphocytes. $x 480$. c) In the submucosal layer is a large lymphatic follicle H\&E. x120. d) Lymphatic follicle with a bright center of reproduction. H\&E x240. 

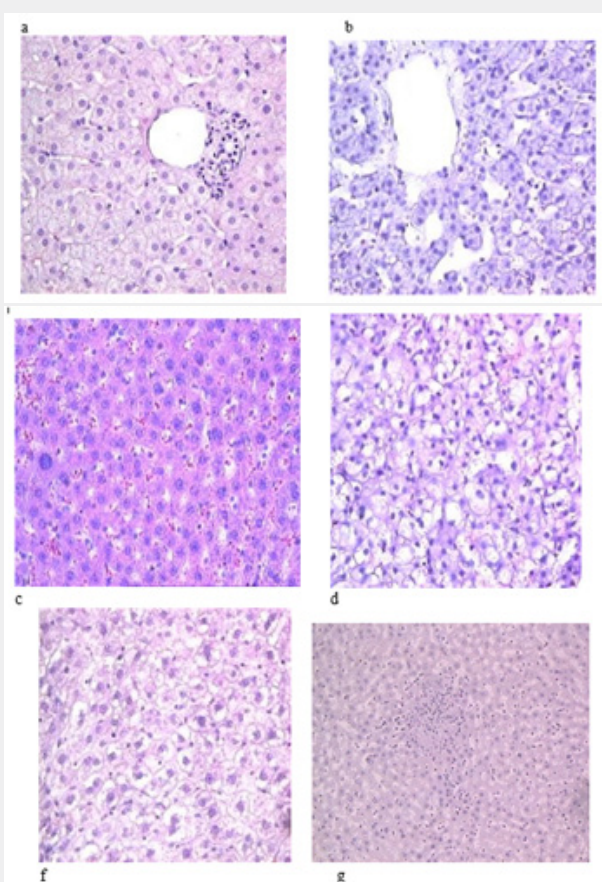

Figure 5 a-g: Liver. coloring H\&E x 480 a) The portal tract is weakly infiltrated by lymphocytes mixed with eosinophils and macrophages. b) Weakly expressed hyperplasia of the cells of the reticuloendothelial system. $x$ 480. c) Dilated central vein. In the lumen of dilated sinusoids, single lymphocytes, red blood cells and eosinophils. Binuclear hepatocytes occur. x480. d) Polymorphism and hyperchromia of hepatocyte nuclei. x480. e) Hydropic degeneration of hepatocytes. x480 f) Infiltrate by lymphocytes x480.

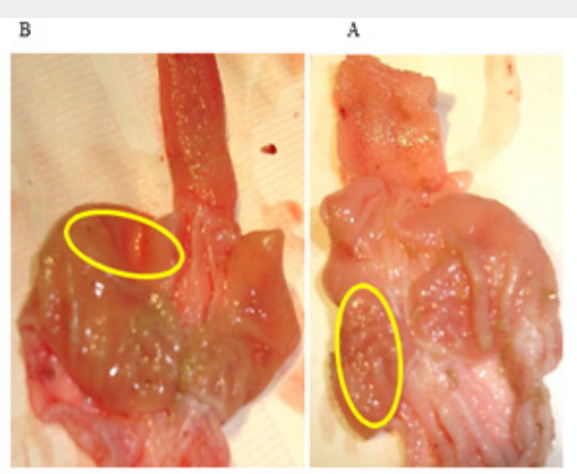

Figure 6: Macrophotography of the gastric mucosa. a) Allocated the mucosa of the stomach body.

b) Selected mucosa antrum.

\section{Results and Discussion}

In animals, after autopsy, the abdominal cavity and mucosa of the gastroduodenal region were reviewed. The spleen and thymus were weighed. The appearance of organs without features. The weight of the spleen ranges from 0.7 to $2 \mathrm{~g}$. In the control of 0.995 g. The weight of the thymus increased by $50-60 \%$.

\section{Morphological Research}

The test material was fixed in 10\% neutral formalin, after histological wiring was poured into paraffin, then sections with a thickness of 4-5 mm were stained with hematoxylin and eosin.
Control group of rats administration of $2.0 \mathrm{ml}$ of saline solution no pathological changes in the body wall, fundal, antral parts of the stomach, duodenum and liver were detected. 2 . Taking into account the proximity of the location and morphological similarity in the description of the morphological picture, we decided to combine the body and the fundal parts of the stomach. It was found that weak or moderate infiltration by lymphoid cells and eosinophils was detected in the muscular and internal plates of the mucous membrane, as well as in the submucosal layer. The number of interepithelial lymphocytes in the superficial, pit and cervical epithelium was not increased in comparison with the 
control group. Often, the submucosal layer revealed edema, dilated, sometimes full-blood vessels. The changes described above were focal in some cases. No changes were detected in the muscular and serous layers of the stomach body wall. When introducing homogenates of the body and fundal divisions in the antral division, no changes were detected, and there is a topographic specificity of the immune response. Group 3: introduction of antral mucosal homogenate. The revealed changes on the parts of the mucous membrane: 1 . in the duodenal mucosa, high intestinal villi covered with a single-layer edged epithelium with the presence of enterocytes with a brush border and goblet cells. The number of interepithelial lymphocytes was increased compared to the control group. There was moderate lymphoplasmacytic infiltration with an admixture of eosinophils, small lymphatic follicles without a center of reproduction in the own plate of the mucous membrane. In addition, mild edema and isolated lymphangiectasia were detected. Inflammatory infiltration often spread to the muscle plate of the mucous membrane and the submucosal layer. In the latter, edema and moderately full - blooded vessels were determined, and in some cases, conglomerates of large lymphatic follicles with light centers of reproduction were determined. 2. there is edema in the antral, fundal, and stomach body, which is more pronounced in the stomach body. 3. in the antral part of the stomach, there was a weakly expressed infiltration by lymphoid cells and eosinophils, which was determined within the muscle and its own MM plates. The pyloric glands had a normal structure. Other layers of the wall in this part of the stomach remained intact. $4^{\text {th }}$ group: were injected with a homogenate of the mucosa of the duodenum. In the duodenal mucosa, high intestinal villi were detected, covered with a single-layer edged epithelium with the presence of goblet cells and enterocytes with a brush border. Inflammatory infiltration often spread [6,7].

\section{Conclusion}

Morphological examination in all the studied parts of the stomach, duodenum and liver showed that there is a hyperplasia of lymphoid tissue. The lymphoid tissue of the digestive system allows us to distinguish three main groups in it: lymphocyteslymphocytes localized in the epithelium and plasma cells that diffusely "populate" their own plate of the mucous

Membrane and lymphoid follicles located in the basal parts of the mucous membrane. At the same time, there is a reaction in the Central organs of the immune system, we found that the weight and size of the spleen and thymus are increased. .Autoimmune pathology is an attack of the immune system against the organs of the "host" and the tissues of its own body, as a result of which their structural and functional damage occurs (figure 3-5), due to immune inflammation. an autoimmune reaction occurs only when the immune system is" tricked " and causes it to attack the cells of its own body. An autoimmune reaction acts on cells of different organs. The formation of antibodies also occurs on the "General" cell structures-the nucleus, mitochondria, and shell, but at the same time we observed specificity in relation to the introduced homogenates. The involvement of several organs in a joint reaction depends on the tolerance of the organ to its own antigens. Reduced tolerance leads to a combined pathology and it is not always possible to identify the "primary" organ that caused the autoimmune reaction. But nevertheless, conducting experimental studies and only under experimental conditions when the animal is sensitized by cells of different organs makes it possible to identify organs with lower or higher tolerance and the presence of joint diseases, such as hepatogenic stomach ulcers. Also, under experimental conditions, we foundthat the reaction to homogenates has a specificity, it was found that the introduction of homogenates of closely located parts of the stomach (fundal and body), the introduction of antrum homogenate causes a reaction in the mucous membrane of the antrum and duodenum, the homogenate of the body mucosa, in the body of the stomach (Figure 6). an Autoimmune reaction occurs only when the immune system is "deceived", which causes it to attack the cells of its own body.

\section{Results}

a) liver damage causes an autoimmune reaction in the mucosa of the gastroduodenal region

b) Damage to the mucous membrane of the gastroduodenal region does not cause an autoimmune reaction in the liver, since it has a high immune tolerance.

c) the Immune response of the macro-organism contributes to the development of autoimmune processes that lead to structural and functional damage to organs and tissues of the body.

d) Autoimmune reaction is specific to cells of different organs and even different parts of the same organ (stomach).

e) at the Same time, the formation of antibodies occurs on the Generals organelle structures of cells.

f) the Involvement of several organs in a joint reaction depends on the local tolerance of the organ to its own antigens.

g) Reduced tolerance leads to a combined pathology, but it is not always possible to identify the "primary" organ that caused the autoimmune reaction.1. liver damage causes an autoimmune reaction in the mucosa of the gastroduodenal region

h) the Involvement of several organs in a joint reaction depends on the local tolerance of the organ to its own antigens.

i) Reduced tolerance leads to a combined pathology, but it is not always possible to identify the "primary" organ that caused the autoimmune reaction.

\section{Summary}

Thanks to the literature data, it is known that the pathogenetic mechanisms of formation of ulcers and erosions in the gastric mucosa in cirrhosis of the liver are not clear and have not been 
clarified. Our research was conducted to establish the pathogenetic mechanisms involved in the formation of damage to the mucous membrane of the gastroduodenal zone.

\section{Goal}

Conduct experimental studies on white rats to identify pathogenetic mechanisms of damage to the gastric and duodenal mucosa. Materials. On 40 white Wistar rats, 2 series of studies were conducted, which convincingly demonstrated that an autoimmune component is present in toxic liver damage, which is combined with the presence of reactive inflammation in the stomach and duodenum. Pre-immunization of animals caused hyperplasia of lymphoid tissue and impaired.

\section{References}

1. Kalinin AV (2002) Symptomatic gastroduodenal ulcers. Clinical lectures on gastroenterology and Hepatology. - Moscow: State Institute of advanced training of doctors of the Ministry of defense of the Russian Federation; Main military clinical hospital named after N. N. Burdenko 1: $283-304$

2. Potashov LV, MA Aliyev, VM Sedov, A Zh Nurmakov (1982) Bleeding from acute and chronic gastroduodenal ulcers. Alma ATA: Kazakhstan, pp. 336.

a. Lapshin AV, Pavlov CHS, VT Ivashkin M (2003) Gastrointestinal bleeding in patients with cirrhosis of the liver. Treatment of cirrhosis of the liver: Method. Recommendations, pp. 4048.

3. Ponomarev AA, Kulikov EP (2003) Unusual ulcers of the stomach and duodenum. Ryazan: Uzorochye, pp. 343.

4. FI Komarov, VA Galkin, AI Ivanov, VA Maksimov (1983) Combined diseases of the organs of the duodeno choledocho pancreatic zone Medicine, p. 256.

5. Trubitsyna IE, Ruchkina IN, Mikhailova SF, Efremov LI, Degterev DA (2018) MALT- system of the gastrointestinal MUCOSA. Experimental and clinical gastroenterology 159(11): 83-86.

6. Trubitsyna IE, Vinokurova LV, Mikhailova ZF, Abdulatipova ZM, Orlova YM, (2019) Gastroenterology the role of the MALT system in the occurrence of immune inflammation of the lungs and pancreas in rats. Saint Petersburg Scientific and practical peer-reviewed journal, Proceedings of the $21^{\text {st }}$ international Slavic-Baltic scientific forum Saint Petersburg-Gastro, 2: 43.

7. Trubitsyna IE, Onishchenko NA, Lundup AV, Knyazev OV, Gulyaev AS (2016) Specific reactivity. Immunomodulatory effect of allogeneic mesenchymal bone marrow stem cells in rats. Experimental and clinical gastroenterology. 11 (135): 59-63.

\section{Your next submission with JuniperPublishers will reach you the below assets}

- Quality Editorial service

- Swift Peer Review

- Reprints availability

- E-prints Service

- Manuscript Podcast for convenient understanding

- Global attainment for your research

- Manuscript accessibility in different formats ( Pdf, E-pub, Full Text, audio)

- Unceasing customer service

Track the below URL for one-step submission https://juniperpublishers.com/online-submission.php 